\title{
Clumpy stellar winds and the obscuration of active galactic nuclei
}

\author{
S. Nayakshin ${ }^{1}$ and J. Cuadra ${ }^{2,3}$ \\ 1 Department of Physics \& Astronomy, University of Leicester, Leicester, LE1 7RH, UK \\ e-mail: sn85@astro.1e.ac.uk \\ 2 Max-Planck-Institut für Astrophysik, Karl-Schwarzschild-Str.1, 85741 Garching, Germany \\ 3 JILA, University of Colorado, 440 UCB, Boulder, CO 80309-0440, USA
}

Received 4 May 2006 / Accepted 15 December 2006

\section{ABSTRACT}

\begin{abstract}
The role of star-formation driven outflows in the obscuration of the central source in Active Galactic Nuclei (AGN) is discussed. The outflow from a sub-parsec scale accretion disc is numerically modelled for parameters appropriate to the Galactic Centre. The resulting obscuration pattern is very patchy, with some lines of sight becoming optically thick to Thomson scattering. A fixed observer would see column depth changing by factors of many over time scales ranging from months to hundreds of years, depending on the physical size of the outflow region. Such winds may be relevant for the obscuration of some AGN, especially "changing look AGN". The winds are Thomson-thick for outflow rates exceeding the Eddington accretion rate limit, which may be satisfied in strong near-AGN starbursts as observed in a few nearby galactic nuclei.
\end{abstract}

Key words. Galaxy: center - accretion, accretion disks - galaxies: active - methods: numerical

\section{Introduction}

According to the simplest version of the unified model of Active Galactic Nuclei (AGN), the central source has fundamentally the same properties in all classes of AGN (e.g., Antonucci \& Miller 1985; Antonucci 1993; Maiolino \& Rieke 1995; Risaliti et al. 1999; Sazonov \& Revnivtsev 2004). It is the orientation of our line of sight with respect to the dusty obscuring environment of an AGN that makes it appear as a type I (relatively un-obscured) or type II object (obscured). Despite all the convincing observational evidence for the existence of the absorber, most frequently thought to be arranged in a shape of a torus, no convincing theoretical model has ever been produced to explain its properties. Recently, Nenkova et al. (2002) and Risaliti et al. (2002) demonstrated that to comply with the small observed torus sizes, the absorber must not be uniform in density but instead rather clumpy, i.e. consisting of individual smaller clouds. Theoretical models of clumpy tori (e.g., Krolik \& Begelman 1986, 1988; Vollmer et al. 2004), however, have all the same fundamental difficulty. As the observations require the torus to be geometrically thick, the velocity dispersion of the cold dusty clouds must be of the order of their orbital velocity in the inner parsec of a galaxy. Optically thick tori would then have clouds colliding at supersonic speeds many times during one orbital period (Krolik \& Begelman 1986, 1988). Therefore, these models have to postulate (somewhat arbitrarily, in our point of view), that the clouds can survive these collisions, behaving in essence as rubber balls.

A warped accretion disc is another potential candidate for the role of the dusty absorber (e.g., Phinney 1989). The disc may be significantly warped by instabilities due to back reaction to mass outflow, or due to the AGN radiation pressure. It may also be warped due to precession in a non-axisymmetric gravitational potential (e.g. Schandl \& Meyer 1994; Pringle 1996; Nayakshin 2005). Finally, there is no reason for an initial disc configuration to be flat if the mass deposition proceeds on a time scale much shorter than the disc viscous time (Phinney 1989), which was probably the case in the Galactic Centre (Nayakshin \& Cuadra 2005; Paumard et al. 2006).

Recently, a number of authors argued that the obscuration may come from winds (e.g., Königl \& Kartje 1994; Kartje et al. 1999; Elvis 2000; Elvis et al. 2004; Elitzur 2005) emanating from the surface of an accretion disk, be these winds driven by line pressure (Proga 2003), continuum radiation pressure, heating, or by hydromagnetic forces. In this paper we consider AGN obscuration by winds driven by star formation activity in an accretion disc. Star or planet formation in AGN discs has been long predicted by theorists (Paczyński 1978; Kolykhalov \& Sunyaev 1980; Lin \& Pringle 1987; Collin \& Zahn 1999; Gammie 2001; Goodman 2003), and recent observations of the young massive stars in the centre of our Galaxy (e.g., Genzel et al. 2003; Ghez et al. 2003) lend a very strong support to this scenario (Levin \& Beloborodov 2003; Nayakshin \& Sunyaev 2005; Paumard et al. 2006). Furthermore, recent mid-infrared observations (e.g., Davies et al. 2006; Mueller Sánchez et al. 2006) of a few nearby galaxies indicate very strong recent star formation activity on scales of few to few tens of parsec from the AGN.

Using the SPH treatment of stellar winds developed by Cuadra et al. $(2005,2006)$ for the stellar winds accreting onto Sgr $\mathrm{A}^{*}$, we calculate the obscuring properties of stellar winds from a starburst in an accretion disc. We find that these winds have very irregular, patchy obscuration patterns. A fixed observer should then see the column depth through the wind varying on time scales from months or years to tens and hundreds of years, depending on the physical size of the wind launching region and the central black hole mass. Such winds can indeed form a dusty and clumpy screen obscuring the AGN emission.

Applicability of these winds to all AGN absorbers is not clear, however. Observations (e.g., Sazonov \& Revnivtsev 2004; Guainazzi et al. 2005) suggest that the structure responsible for AGN obscuration should be optically thick over a large fraction (one third) of the sky seen from the central engine. Our models 
can achieve such a high average line of sight column depth only if the mass loss rate in the wind is super-Eddington. While such high mass outflow rates are not impossible for a short period of time in the life of a bright AGN/quasar (e.g., Davies et al. 2006), it would be rather remarkable if most local AGN, having low luminosities by quasar standards (e.g., Fig. 1.9 in Ho 2005), were powering such strong outflows.

\section{Stellar winds from AGN star-forming discs}

AGN discs are expected to be massive enough and become selfgravitating at large distances $(\gtrsim 0.01-0.1 \mathrm{pc})$ from the supermassive black holes (SMBH). If the disc cooling time is short, gravitational collapse and formation of stars or even planets is predicted (e.g., Paczyński 1978; Kolykhalov \& Sunyaev 1980; Shlosman \& Begelman 1989; Gammie 2001). Young massive stars in the central parsec of our own Galaxy were most likely created in this way (Paumard et al. 2006), apparently with a significant over-abundance of high mass stars (Nayakshin \& Sunyaev 2005) compared to "standard" star formation. As stars are born from the accretion disc, they launch powerful radiation fields and stellar winds, some of which will break through the disc. Low mass proto-stars may prove equally effective in launching outflows in these circumstances, as the rate at which a proto-star captures gas from the disk is typically superEddington (Nayakshin 2006). In addition, when the gas in the disk is depleted to about half of its initial surface density, the stellar velocity dispersion starts to grow, and soon the stellar disc becomes thicker than the gas disc (Nayakshin 2006). Stellar winds can then escape from the disc directly.

We attempt to simulate this complex situation in a simplified setting, concentrating only on the wind part of it. For this reason we do not include the gas in the negligibly thin and flat accretion disc from which the stars are born, assuming that it occupies a fixed plane $(z=0)$. To capture some of the expected diversity of the stellar populations and conditions in this problem, the stars are divided into two groups. The first produces winds with velocities $v_{\mathrm{w}}=300 \mathrm{~km} \mathrm{~s}^{-1}$, whereas the second has $v_{\mathrm{w}}=700 \mathrm{~km} \mathrm{~s}^{-1}$. Both types of stars have mass loss rates of $2.5 \times 10^{-4} M_{\odot}$ year $^{-1}$, and are situated in a flat circularly rotating Keplerian disk of geometrical thickness $H(R)=0.1 R$, where $R$ is the radius ${ }^{1}$. In total, we have 200 mass shredding stars, thus amounting to the mass loss rate of $0.1 M_{\odot}$ year $^{-1}$, which is a factor of few superEddington for a SMBH mass of $M_{\mathrm{BH}}=3.5 \times 10^{6} M_{\odot}$. The disc inner and outer radii are $R_{\text {in }}=1.5$ and $R_{\text {out }}=8$, respectively. The stellar surface density follows the law $\Sigma_{*}(R) \propto R^{-1}$. The initial phase (i.e. the $\phi$-coordinate) of the stars in the disk is generated randomly, as is the vertical coordinate within the $-H$ to $+H$ limits. Initially, the computational domain is filled with hot tenuous gas with velocity greater than the escape velocity. This gas quickly outflows from the region. At the end of the simulation the stellar winds exceed the initial gas mass by a factor of few tens.

To perform simulations, we use the SPH code GADGET-2 (Springel 2005) modified to model stellar winds as new gas particles ejected from the stars. This method was described and thoroughly tested by Cuadra et al. (2005, 2006).

A snapshot of the simulation is shown in Fig. 1 at time $t \approx 2200 \mathrm{yr}$ after the beginning of the simulation, which corresponds to $\sim 36$ dynamical times at $R=1$. While there is no true steady state for a system of a finite number of moving stars, the

1 The unit of length used here is 1 arcsecond, which corresponds to about $1.2 \times 10^{17} \mathrm{~cm}$ or $0.04 \mathrm{pc}$ at the Galactic Centre distance of $8 \mathrm{kpc}$. snapshot is fairly typical of the morphology of the stellar wind. The face-on view (left panel) shows that some of the shocked wind managed to cool down and formed a small-scale disc. The disc formation occurs at the inner part of the computational domain, because the stellar wind density is highest there, leading to shocks, thermalization and rapid cooling, with the frequent collisions of gaseous clumps "wasting" the momentum of the winds. Moreover, the escape velocity from the region is around $600 \mathrm{~km} \mathrm{~s}^{-1}$, i.e. higher than initial velocity of the slower winds. With time the disc grows radially to both smaller and larger radii. However, the formation of the disc (cf. Cuadra et al. 2006) is physically significant only if the gaseous accretion disc from which the stars were born is entirely absent, as is the case presently in the Galactic Centre. Otherwise, the cooled disc and the "spiral arms" seen at intermediate radii would simply blend in with the much more massive underlying gas accretion disc. Notice that the gas disc is slightly inclined with respect to the orbital plane of the stellar disc. This is the result of the angular momentum of the gas originally present in the computational domain.

Contrary to the frequent collisions and high escape velocity in the inner region, the conditions at larger radii allow direct escape of both the fast diffuse and the slower cooler clumpy winds. Due to the final extent of the stellar disc and a projection effect, the wind morphology reminds and "X"-shape (Fig. 1, right panel).

The edge-on view (right panel) of the stellar disc also shows that the plane of the gas disc at $R \lesssim 2$ is very slightly tilted with respect to the orbital plane of the stellar disc. This is an artefact of the initial conditions.

The left panel of Fig. 2 shows the obscuring column depth of the winds as seen from the SMBH. Since we concentrate on the obscuration from the wind and not from the disc, we only plot gas with radial distances $R \gtrsim 1.6$ from the SMBH (see however Sect. 4 and Fig. 3).

Notice the very irregular patchy structure of the (yellow) optically thicker regions. The contrast between those and neighbouring less dense patches of sky is frequently a factor of 10 or more. Since the winds are rotating at a fraction of the local Keplerian angular frequency, $\Omega$, this implies that the column depth sampled by the observer will vary on time scales as short as $\sim 10^{-3}-10^{-2}$ of $1 / \Omega$, i.e., $t_{\mathrm{var}} \sim$ $0.04-0.4$ year $(r / 0.1 \mathrm{pc})^{3 / 2} M_{8}^{-1 / 2}$. Here $r$ is radius $R$ in physical units. The dotted pattern at the $\cos \theta=0$ plane are the dense regions of stellar winds immediately next to the stars.

The right panel of Fig. 2 shows the isotropic mass loss rate along the line of sight, defined as

$\dot{M} \equiv \frac{\int \mathrm{d} \Sigma 4 \pi r^{2} \rho v_{R}}{\int \mathrm{d} \Sigma}$,

where the integral is taken along the line of sight defined by a given $\theta$ and $\phi$. This quantity is useful for comparison with observations of AGN outflows. An observer has only one line of sight available at any given moment, and the assumption frequently made is that the outflow is roughly isotropic, thus $\dot{M} \sim 4 \pi r^{2} \rho v_{R}$. We find that there are lines of sight that yield isotropic mass outflow rates of $\sim 1-10 M_{\odot}$ year $^{-1}$, which is as much as a hundred times larger than the correct sky-averaged value. Also note that not all of the optically thicker regions seen in the left panel of Fig. 2 appear equally prominently in the outflow map in the right panel of the figure, as some of these structures have a small radial velocity or are even infalling to smaller radii. 

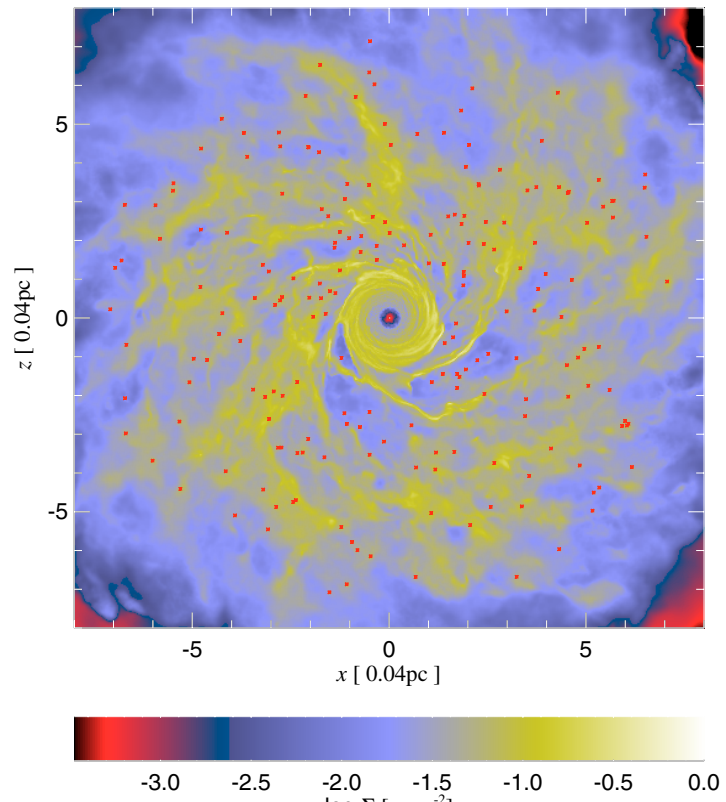
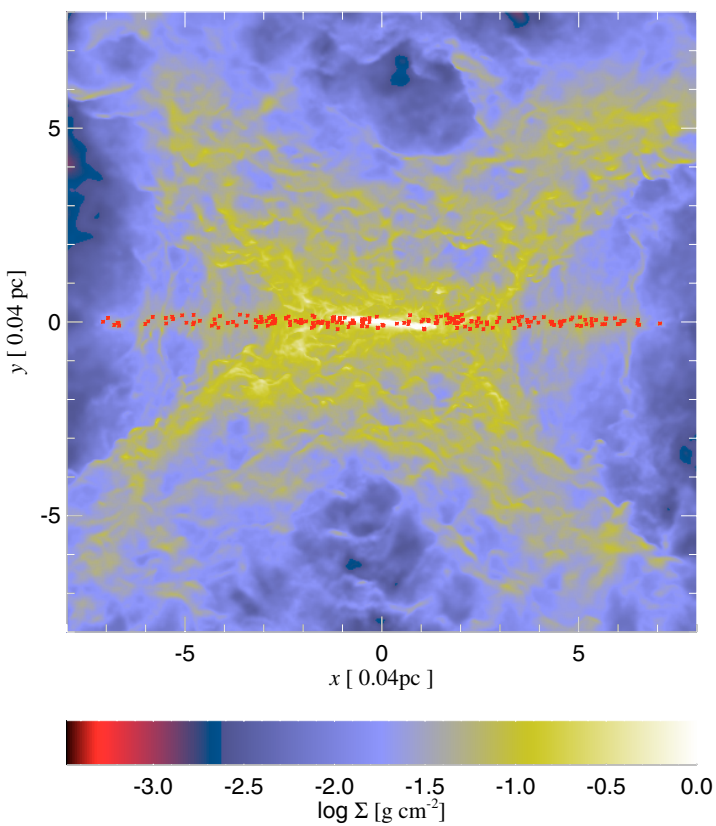

Fig. 1. Face-on (left) and edge-on (right) views of the simulation domain for the flat stellar disc configuration. Red asterisks show the location of the wind-producing stars used in the simulation (the red circle in the centre of the left panel is not a star, but a low column depth region centred on the SMBH).
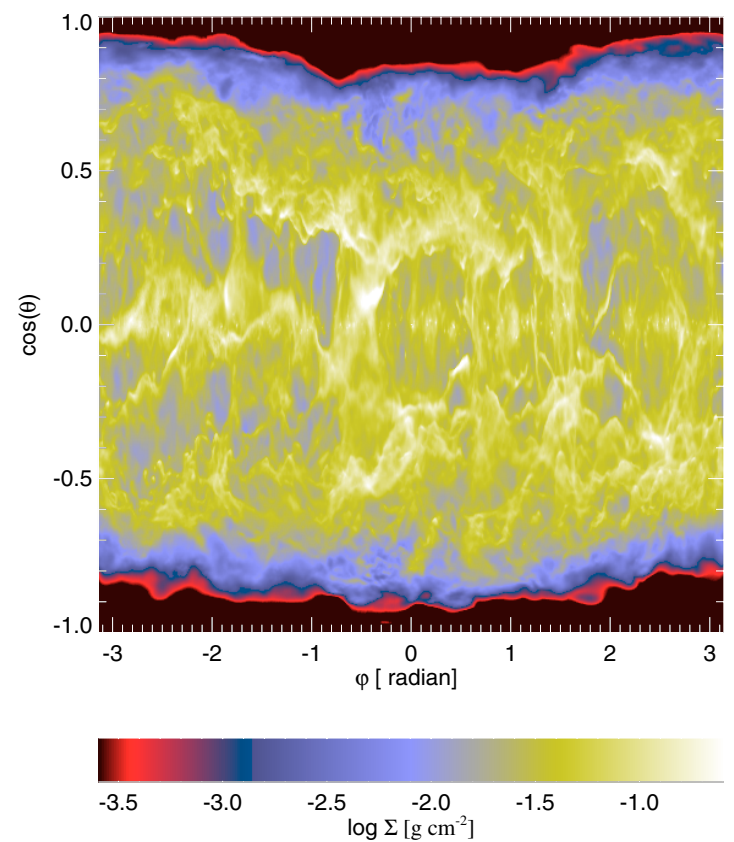

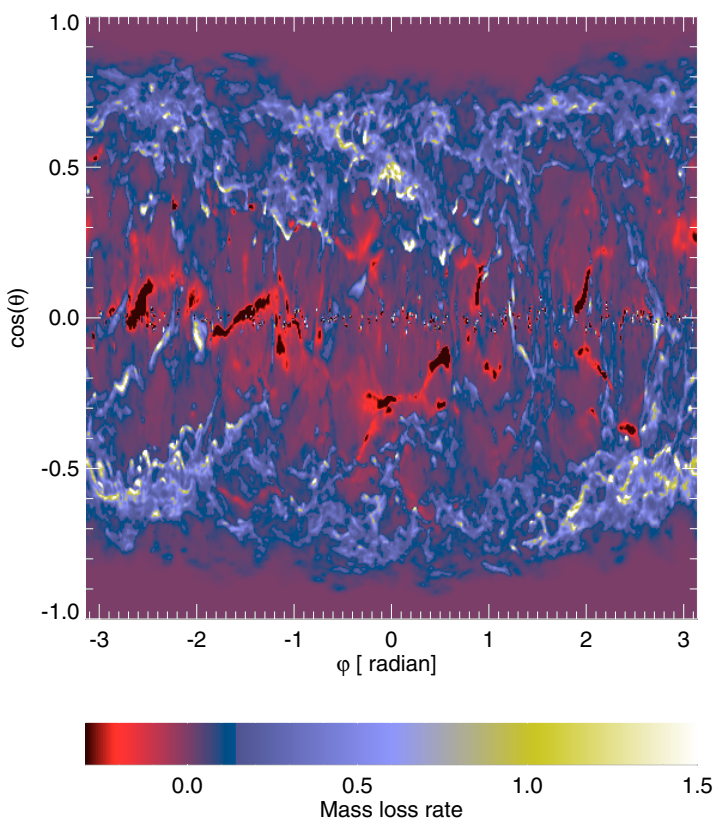

Fig. 2. Column depth through the wind (left) and isotropic mass outflow rate (right; in units of $M_{\odot}$ year ${ }^{-1}$ ) for the simulation shown in Fig. 1, as seen from an observer located at the SMBH. Note the large variations of the obscuring column depth over small angular scales. The gaseous inner disc seen in the left panel of Fig. 1 has been excluded.

\section{Comparison to observations}

Recent multi-wavelength observations (e.g., Jaffe et al. 2004; Packham et al. 2005; Prieto et al. 2005) indicate that AGN absorbers are relatively small, parsec-scale structures, rather than extended (100 to 1000 parsec) as was thought a decade earlier on the basis of observations with poorer angular resolution. Nenkova et al. (2002) and Elitzur (2005) demonstrate that this observational fact demands the "torus" to be clumpy in order to allow high and low temperature regions to be simultaneously present at the same location in the torus. Nenkova et al. (2002) show that the average number of clouds on the line of sight to the nucleus, $N_{\mathrm{a}}$, should be of the order of a few to ten. Let $M_{\mathrm{c}}$ be the mass of such a cloud, $M_{\mathrm{c}}=\Sigma_{\mathrm{c}} \pi r_{\mathrm{c}}^{2}$, and $\Sigma_{\mathrm{c}}$ and $r_{\mathrm{c}}$ be the surface density and cloud radius, respectively. In the model of these authors, the average column depth on the line of sight, $N_{\mathrm{a}} \Sigma_{\mathrm{c}}$ is of the order of $1 \mathrm{~g} \mathrm{~cm}^{-2}$ or larger, which is also reasonable given the large fraction of AGN that are Thomson-thick (e.g., Sazonov \& Revnivtsev 2004; Guainazzi et al. 2005). The total number of the clouds, $N_{\mathrm{t}}$, is connected to $N_{\mathrm{a}}$ through

$N_{\mathrm{a}}=\frac{N_{\mathrm{t}} \pi r_{\mathrm{c}}^{2}}{2 \pi r_{\mathrm{t}}^{2}}$ 

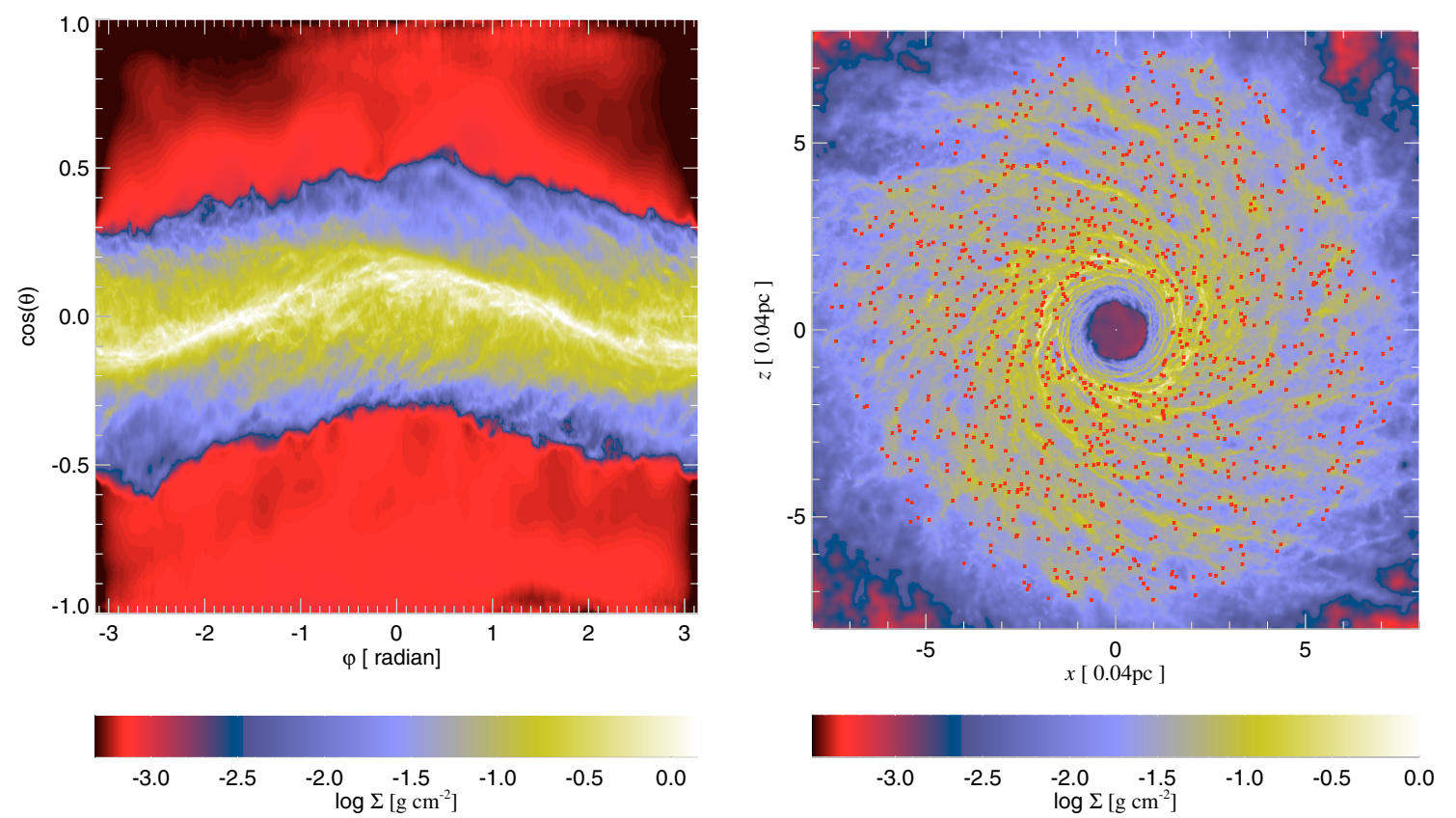

Fig. 3. Left panel: same as that of Fig. 2 but for the super-massive black hole mass of $10^{8} M_{\odot}$ and mass loss rate of $0.5 M_{\odot}$ year ${ }^{-1}$. Note that the outflow is concentrated towards the stellar disc midplane and does not cover a large solid angle. The cause of the warp in the figure is the initial hot gas present in the computational domain; it is physically unimportant as discussed in the text explaining Fig. 4. Right panel: pole-on view of this simulations.

where $r_{\mathrm{t}}$ is the typical radius of the torus, and we assumed that it covers roughly $2 \pi$ of the sky as seen from the SMBH. Now, assume that the torus is made up of clouds that form in the outflowing disk wind, as in the simulations presented in this paper. The total mass outflow rate of the clouds is then

$\dot{M}_{\text {wind }} \sim 2 \pi r_{\mathrm{t}} N_{\mathrm{a}} \Sigma_{\mathrm{c}} v_{\mathrm{K}} \sim 15 \frac{M_{\odot}}{\text { year }} N_{\mathrm{a}} \Sigma_{\mathrm{c}}\left(\frac{r_{\mathrm{t}}}{1 \mathrm{pc}}\right)^{1 / 2} M_{8}^{1 / 2}$,

where $M_{8}$ is the SMBH mass in units of $10^{8} M_{\odot}$, and we assumed that clouds radial velocities are of the order of the local Keplerian speed, $v_{\mathrm{K}}$. Now, the Eddington accretion rate is $4 \pi G M_{\mathrm{BH}} m_{\mathrm{p}} / \epsilon c \sigma_{\mathrm{T}} \approx 2 M_{\odot}$ year $^{-1} M_{8}$. Thus the required wind mass loss rate (Eq. (3)) is an order of magnitude higher, typically, than the Eddington accretion rate for a $M_{8} \sim 1$ object and a parsec-scale torus. The estimate of $\dot{M}_{\text {wind }}$ could be reduced somewhat by postulating even smaller torus sizes, but it is hard to make $r_{\mathrm{t}}$ much smaller than $\sim 0.1 \mathrm{pc}$ as then the dust would be sublimated by the AGN radiation field (e.g., Eq. (3.2) in Emmering et al. 1992). However, we rather think that our estimate (Eq. (3)) is too optimistic, i.e., that even higher mass outflow rates are needed, as the observations and modelling of optically thick sources require a minimum absorbing column depth, and hence in a good fraction of sources $N_{\mathrm{a}} \Sigma_{\mathrm{c}}$ may be actually much higher than $1 \mathrm{~g} \mathrm{~cm}^{-2}$.

Considering a specific case of the local obscured AGNs studied by Guainazzi et al. (2005), we note that the bolometric luminosities of these objects in the infrared, X-ray and optical bands are in the range $L \sim 10^{43}-$ few $\times 10^{44} \mathrm{erg} / \mathrm{s}$, which implies SMBH accretion rates of "only" $\sim 0.01 M_{\odot}$ year $^{-1}$ for the standard radiative efficiency. Hence if the obscuration of the optically thick objects in that sample were provided by the winds, we would conclude that the SMBH accretion process must be very wasteful, with 100-10 000 times more mass flowing out of the inner parsec than accreting on the SMBH (note that these moderately bright AGN are not likely to be in the non-radiative accretion flow regime when vigorous outflows are in fact expected, e.g.,
Blandford \& Begelman 1999). It would also require a very high mass influx into the inner parsec to sustain such winds. Given the difficulty of delivering enough fuel to the SMBHs even in the earlier gas-rich epochs (Thompson et al. 2005), it is hard to see how such high mass influxes could be maintained in the local AGN.

We now estimate the ionisation parameter $\left(\xi \equiv L_{\mathrm{x}} /\left(n_{\mathrm{H}} r^{2}\right)\right.$, where $n_{\mathrm{H}}$ is hydrogen density in $\left.\mathrm{cm}^{-3}\right)$ of the gas in the wind illuminated by AGN with X-ray luminosity of $L_{\mathrm{X}}=10^{43} L_{43} \mathrm{erg} / \mathrm{s}$. Let us first assume that the wind is uniform in density. In that case, $\xi \sim 20 L_{43} v_{8}\left(20 M_{\odot}\right.$ year $\left.^{-1}\right) /\left(\dot{M}_{\text {wind }}\right)$. The recombination time to establish ionisation equilibrium is well under a year (e.g., rescale Eq. (2) from Nayakshin \& Kazanas 2002), much shorter than the outflow time scale, thus ionisation equilibrium will be quickly set. Now, if the winds are clumpy, then within the clumps the gas density is higher by few orders of magnitude than we assumed here, and thus $\xi$ would be much lower inside the clumps. It is then natural to expect that the dense regions of the wind will be cool and dusty.

While we concentrated on simulations for the Galactic Centre conditions, i.e. for a relatively light-weight black hole, we have also explored models for a $M_{\mathrm{BH}}=10^{8} M_{\odot}$, which should be more relevant for distant brighter AGN. If the winds emanate from the same physical region as we considered here, i.e. a small fraction of a parsec, then the results are less promising than they are for the GC conditions. For higher SMBH mass, the escape velocity is larger at the same physical distance from the black hole $\left(v_{\mathrm{esc}} \propto \sqrt{M_{\mathrm{BH}} / r}\right)$. Obviously then, stellar winds with $v_{\mathrm{w}} \ll v_{\text {esc }}$ are disc-hugging, more like a corona to a disc. Figure 3, left panel, shows the obscuring column depth of such a simulation with the total stellar wind loss of $0.5 M_{\odot}$ year $^{-1}$, and same geometrical parameters of stellar disc, though now with 1000 stellar wind sources. As seen from the figure, the obscuration from stellar winds is concentrated to lines of sight close to the disc, and thus cannot account for the observed large geometrical scale height of the absorber. Stellar winds emitted 


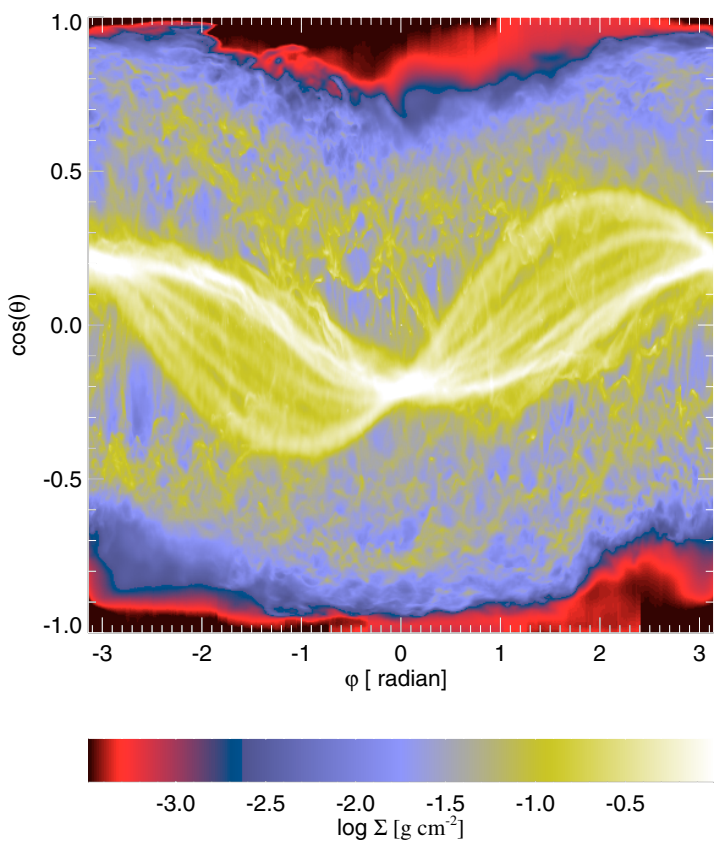

Fig. 4. Same as the left panel of Fig. 2 but for slightly different initial conditions and with the cold tilted and warped inner disc included in the figure. Notice that this very light (only $\sim 3 M_{\odot}$ ) disc is a very efficient absorber.

farther out, at, say, $r \sim 1-10$ parsec distance would be more geometrically thick, but then these sizes may be at odds with the small tori implied by observations (e.g., Elitzur 2005). The right panel of the figure shows the pole-on view of the simulation.

\section{Discussion and conclusions}

Accreting black holes can drive strong outflows via X-ray heating, line or continuous radiation pressure, and hydromagnetic forces(e.g., Begelman et al. 1983; Königl \& Kartje 1994; Kartje et al. 1999; Proga 2003). Star formation on the outskirts of cool massive accretion discs will also result in winds driven by outflows from the young massive stars (Cuadra et al. 2005). Here we studied the obscuration properties of the AGN outflows of the latter type. We found that these outflows are quite clumpy, with the lines of sight passing through the clumps becoming moderately optically thick to Thomson scattering for high enough wind mass loss rates. In particular, to be consistent with observational (e.g., Guainazzi et al. 2005) and radiative transfer modelling constraints (e.g., Nenkova et al. 2002; Elitzur 2005), the outflow rates should be as large as $1-100 M_{\odot}$ year $^{-1}$, which is probably hard to satisfy for the local AGN that are likely to be accreting at only $0.01-0.1$ of the Eddington accretion rates (and even lower if we also consider Low Luminosity AGN; e.g., Ho 2005). On the other hand, some nearby galaxies show signs of recent vigorous star formation activity in the central parsecs (Davies et al. 2006; Mueller Sánchez et al. 2006) that could possibly power such outflows.

One limitation of our work is that we have not considered here supernova explosions of massive stars. If these occur frequently enough, and if the blast waves are loaded by surrounding cool gas from the disc, then significant obscuration may result from supernova explosions, as shown by Wada \& Norman (2002). More quantitative work is required to test plausibility of supernova-heated torus, in particular for lower luminosity sources, as then one has two opposite constraints. Namely, due to the discrete nature of energy release in supernova explosions, it is desirable to have many explosions during a dynamical time, $\sim 100$ years. At the same time, the observed AGN luminosity sets the upper limit on number of such explosions.

It is not possible to simulate or critically assess all the torus models in this paper, but we feel that all outflow obscuration models will face the same basic problem - the requirement of too much mass outflux even in low luminosity sources (Eq. (3)). For example, consider the recent "wind-torus" model of Elitzur \& Shlosman (2006) for the source NGC 3079. The authors conclude that mass outflow rates of only $\sim 0.02 M_{\odot}$ year $^{-1}$ would be sufficient to explain the obscuration in this source. To reach this conclusion, they estimate the absorber column depth to be $\sim 10^{23}$ hydrogen atoms $\mathrm{cm}^{-2}$, and the outflow velocity to be around $\sim 10 \mathrm{~km} \mathrm{~s}^{-1}$. However, the column depth of $\sim 10^{23}$ hydrogen atoms $\mathrm{cm}^{-2}$ would be at least an order of magnitude too thin for Compton-thick sources. Further, we note that escape velocity from the region considered is about $300 \mathrm{~km} \mathrm{~s}^{-1}$. It is not clear how these very slow winds may be outflowing at all in this situation. In fact even the original quasi-static torus model by Krolik \& Begelman (1986) assumed much larger velocity dispersion in gas motions. Henceforth, Elitzur \& Shlosman (2006) estimate, given in their Eq. (1), should be increased by a factor of at least 100 for winds in Compton-thick sources, bringing it much closer to our estimate given in Eq. (3).

Nevertheless, we would argue that AGN winds are important for obscuration in possibly all sources, but in spectral regions that do not require high obscuring column depth, i.e. in soft X-rays or in the UV. An exception to this would be the most luminous quasars where winds may be optically thick across the whole spectrum. In a garden-variety AGN, however, it is likely that AGN winds work in concert with an additional optically thick absorber. One candidate for this is a warped accretion disc, as discussed in the Introduction. To illustrate the point, we present one additional simulation which is exactly identical to the one shown in Fig. 1, except for the initial conditions: the simulated domain was initially filled with hot gas at a higher density. While the results were very similar to those presented earlier in the paper, one difference was that the gas disc formed by the cooled fall-back material was tilted out of the symmetry plane due to interaction between the winds and the gas present in the computational domain initially. Figure 4 shows the obscuration pattern of this simulation in the same way as in the left panel in Fig. 2, but including the tilted and slightly warped gas disc. As Fig. 4 shows, the obscuration provided by the winds literally pales in comparison with that produced by the warped disc, whose mass is only $\sim 3 M_{\odot}$. Obscuration by a warped accretion disc requires no continuous mass or energy input, and would work for low or high luminosity AGN as long as the discs are sufficiently massive and strongly warped.

Acknowledgements. The authors acknowledge discussions and comments from Ken Pounds and Sergei Sazonov.

\section{References}

Antonucci, R. 1993, ARA\&A, 31, 473

Antonucci, R. R. J., \& Miller, J. S. 1985, ApJ, 297, 621

Begelman, M. C., McKee, C. F., \& Shields, G. A. 1983, ApJ, 271, 70

Blandford, R. D., \& Begelman, M. C. 1999, MNRAS, 303, L1

Collin, S., \& Zahn, J. 1999, A\&A, 344, 433

Cuadra, J., Nayakshin, S., Springel, V., \& Di Matteo, T. 2005, MNRAS, 360, L55

Cuadra, J., Nayakshin, S., Springel, V., \& Di Matteo, T. 2006, MNRAS, 366, 358

Davies, R. I., Thomas, J., Genzel, R., et al. 2006, ApJ, 646, 754 
Elitzur, M. 2005, To appear in the proceedings of "QSO Hosts: Evolution and Environment", Leiden, August 2005 [arXiv: astro-ph/0512025]

Elitzur, M., \& Shlosman, I. 2006, ApJ, 648, L101

Elvis, M. 2000, ApJ, 545, 63

Elvis, M., Risaliti, G., Nicastro, F., et al. 2004, ApJ, 615, L25

Emmering, R. T., Blandford, R. D., \& Shlosman, I. 1992, ApJ, 385, 460

Gammie, C. F. 2001, ApJ, 553, 174

Genzel, R., Schödel, R., Ott, T., et al. 2003, ApJ, 594, 812

Ghez, A. M., Duchêne, G., Matthews, K., et al. 2003, ApJ, 586, L127

Goodman, J. 2003, MNRAS, 339, 937

Guainazzi, M., Matt, G., \& Perola, G. C. 2005, A\&A, 444, 119

Ho, L. C. 2005, Ap\&SS, 300, 219

Jaffe, W., Meisenheimer, K., Röttgering, H. J. A., et al. 2004, Nature, 429, 47

Kartje, J. F., Königl, A., \& Elitzur, M. 1999, ApJ, 513, 180

Kolykhalov, P. I., \& Sunyaev, R. A. 1980, Soviet Astron. Lett., 6, 357

Königl, A., \& Kartje, J. F. 1994, ApJ, 434, 446

Krolik, J. H., \& Begelman, M. C. 1986, ApJ, 308, L55

Krolik, J. H., \& Begelman, M. C. 1988, ApJ, 329, 702

Levin, Y., \& Beloborodov, A. M. 2003, ApJ, 590, L33

Lin, D. N. C., \& Pringle, J. E. 1987, MNRAS, 225, 607

Maiolino, R., \& Rieke, G. H. 1995, ApJ, 454, 95

Mueller Sánchez, F., Davies, R. I., Eisenhauer, F., et al. 2006, A\&A, 454, 481
Nayakshin, S. 2005, MNRAS, 359, 545

Nayakshin, S. 2006, MNRAS, 372, 143

Nayakshin, S., \& Kazanas, D. 2002, ApJ, 567, 85

Nayakshin, S., \& Cuadra, J. 2005, A\&A, 437, 437

Nayakshin, S., \& Sunyaev, R. 2005, MNRAS, 364, L23

Nenkova, M., Ivezić, Z., \& Elitzur, M. 2002, ApJ, 570, L9

Packham, C., Radomski, J. T., Roche, P. F., et al. 2005, ApJ, 618, L17

Paczyński, B. 1978, Acta Astron., 28, 91

Paumard, T., Genzel, R., Martins, F., et al. 2006, ApJ, 643, 1011

Phinney, E. S. 1989, in Theory of Accretion Disks, NATO ASIC Proc., 290, 457

Prieto, M. A., Maciejewski, W., \& Reunanen, J. 2005, AJ, 130, 1472

Pringle, J. E. 1996, MNRAS, 281, 357

Proga, D. 2003, ApJ, 585, 406

Risaliti, G., Maiolino, R., \& Salvati, M. 1999, ApJ, 522, 157

Risaliti, G., Elvis, M., \& Nicastro, F. 2002, ApJ, 571, 234

Sazonov, S. Y., \& Revnivtsev, M. G. 2004, A\&A, 423, 469

Schandl, S., \& Meyer, F. 1994, A\&A, 289, 149

Shlosman, I., \& Begelman, M. C. 1989, ApJ, 341, 685

Springel, V. 2005, MNRAS, 364, 1105

Thompson, T. A., Quataert, E., \& Murray, N. 2005, ApJ, 630, 167

Vollmer, B., Beckert, T., \& Duschl, W. J. 2004, A\&A, 413, 949

Wada, K., \& Norman, C. A. 2002, ApJ, 566, L21 